\title{
WELL-POSEDNESS AND SLIDING MODE CONTROL
}

\author{
TUlLio ZOLEZZI ${ }^{1}$
}

\begin{abstract}
Sliding mode control of ordinary differential equations is considered. A key robustness property, called approximability, is studied from an optimization point of view. It is proved that Tikhonov well-posedness of a suitably defined optimization problem is intimately related to approximability. Making use of this link, new approximability criteria are obtained for nonlinear sliding mode control systems.
\end{abstract}

Mathematics Subject Classification. 49K40, 93B12.

Received February 6, 2004.

\section{INTRODUCTION}

A nonlinear control system described by ordinary differential equations with a prescribed sliding manifold is considered on a bounded time interval. Sliding mode control aims at driving and keeping the system state vector on the sliding manifold by suitable feedback control laws. A number of significant control problems can thereby solved in an effective way, among them invariance to process dynamics characteristics, disturbance decoupling, stabilization, tracking control and model following, control under uncertainty. We refer to $[6,9,12,14]$ for overviews of the underlying theory and applications.

A basic problem in this area is the following. Suppose that the sliding condition is approximately fulfilled. Is it then true that the dynamical behavior of the (so called) real states of the system approaches the ideal sliding behavior as the imperfections preventing ideal sliding disappear?

The previous question deals with robustness features of the sliding mode control methodology. In the applications of sliding mode control, the real states, namely those which fulfill only approximately the sliding condition, are of interest due to unavoidable imperfections, small errors in the control process, small delays etc. If a sliding mode control system is not robust in the previous sense, then very small errors (of any nature) may destroy good performance of the given system.

According to the terminology of [14], ideal states are those fulfilling exactly the sliding condition, real states are those fulfilling only approximately such a state constraint, and any reason preventing ideal sliding is called a nonideality. A sliding mode control system fulfills the approximability property (see [3]) provided all real states converge towards the unique sliding state as the nonidealities disappear (see the next section for the complete definitions).

The problem is considered in [14], Chapter 2, for dynamics affine in the control law, and is put on a mathematically rigorous basis in [3] for non linear dynamics. Sufficient conditions guaranteeing that all real states

Keywords and phrases. Sliding mode control, Tikhonov well-posedness, approximability.

1 Dipartimento di Matematica, via Dodecaneso 35, 16146 Genova, Italy; zolezzi@dima.unige.it 
converge toward a well-defined sliding state as the nonidealities disappear are obtained in $[3,4]$, thereby yielding approximability.

In this paper we propose a new and more appropriate definition of real states than the one used in [3,4], moreover we consider the previous problem from an optimization point of view.

Real states arise due to imperfections (of any nature) acting on the sliding phase of the control process, corresponding to possibly discontinuous feedback control laws. It follows that the real states are mathematically described by classical (i.e. almost everywhere), or Filippov solutions to the ordinary differential equations modeling the control system. For the notion and the relevant properties of Filippov solutions see $[10,11]$. See [14] for showing why Filippov solutions are adequate in this context. We take into account both possibilities by enlarging in such a way the definition of real states. The previous approximability concepts, we considered in [3] (Def. 3, p. 54) and in [4], were based on defining real states as classical solutions. No Filippov solutions were considered in this context. Imposing convergence of the real states, as defined in the present paper, in a broader sense than in $[3,4]$, strengthens the corresponding definition of approximability as compared with the previous setting. Hence the known criteria guaranteeing approximability are not necessarily valid in this new setting. We shall however show that the basic sufficient conditions yielding approximability generalize to the new framework.

Within this new approach, we show in Section 3 that approximability is equivalent to Tikhonov well-posedness (see [8], Chap. I) of a suitably defined optimization problem, whose minimizer is the sliding state. Roughly speaking, the real states correspond to the minimizing sequences of such optimization problem, hence their convergence is at issue, whence the link with well-posedness. In Section 4 we obtain new approximability criteria, which substantially extend the known conditions, making use of the link with Tikhonov well-posedness. Moreover we show that approximability can be characterized in terms of the (suitably measured) gap between system states and the sliding trajectory.

The results of this paper show that an approach to the approximability problem for sliding mode control systems, based on optimization, allows us to obtain a better insight and more general results. Moreover the same approach could be useful in understanding approximability properties of second (and higher) order sliding mode control methods (see e.g. [2]), currently under development.

\section{Problem statement}

We consider a sliding mode control system with state variable $x \in R^{N}$, control variable $u \in R^{K}$, given by

$$
\begin{gathered}
\dot{x}=f(t, x, u), u \in U, 0 \leq t \leq T ; x(0)=\bar{x} ; \\
s(t, x)=0 .
\end{gathered}
$$

The dynamics

$$
f:[0, T] \times R^{N} \times U \rightarrow R^{N}
$$

are Carathéodory functions, $U$ is a closed subset of $R^{K}, \bar{x} \in R^{N}$, and

$$
s:[0, T] \times R^{N} \rightarrow R^{M}
$$

is continuous.

Given $\bar{x} \in R^{N}$, we consider as admissible control laws in (2.1) all functions

$$
u:[0, T] \times R^{N} \rightarrow U
$$

which are $L \otimes B$-measurable, i.e. measurable with respect to the $\sigma$-algebra generated by the products of the Lebesgue measurable subsets of $[0, T]$ and the Borel measurable subsets of $R^{N}$, and which fulfill the following 
property. For any such $u$, if the differential system in (2.1) has a classical (i.e. almost everywhere), or a Filippov solution $x$ on $[0, T]$ for any $\bar{x}$, then $u[\cdot, x(\cdot)] \in L^{\infty}(0, T)$. We denote by $U_{\infty}$ the set of all admissible controls.

Given $\bar{x}$, we denote by $W(\bar{x})$ the set of all absolutely continuous functions $x$ satisfying (2.1) corresponding to some $u$ belonging to $U_{\infty}$ either in the a.e. or in the Filippov sense. To simplify the notations, we shall write

$$
\dot{x}=f(t, x, u) \text { on }[0, T]
$$

meaning that $u \in U_{\infty}$ and $x$ is either a classical of Filippov solution on $[0, T]$. The $L^{\infty}(0, T)$ norm of $v$ is denoted by $\|v\|_{\infty}$.

The control system (2.1), (2.2) fulfills the (first order) approximability property if the following properties (A), (B) are true.

(A) For every $\bar{x} \in R^{N}$ such that $s(0, \bar{x})=0$ there exists a unique sliding state $y$ issued from $\bar{x}$, namely $y$ is absolutely continuous on $[0, T]$ and

$$
\begin{aligned}
& y(0)=\bar{x}, s[t, y(t)]=0 \text { for every } t \in[0, T], \\
& \dot{y}=f\left(t, y, u^{*}\right) \text { on }[0, T] \text { for some } u^{*} \in U_{\infty} .
\end{aligned}
$$

(B) For every sequences $u_{n} \in U_{\infty}$ and $x_{n}$ such that

$$
\sup \left\|u_{n}\left[\cdot, x_{n}(\cdot)\right]\right\|_{\infty}<+\infty, \dot{x}_{n}=f\left(t, x_{n}, u_{n}\right) \text { on }[0, T], x_{n}(0) \rightarrow \bar{x},
$$

and

$$
s\left(t, x_{n}\right) \hookrightarrow 0
$$

we have

$$
x_{n} \hookrightarrow y
$$

By $\hookrightarrow$ we denote uniform convergence on $[0, T]$.

The definition of approximability presented here is an extension of that in [3]: we consider a broader set of admissible controls, and the existence of the equivalent control is not required. Moreover it is a modification of that in [4], where existence of the equivalent control was not required and Hermes solutions were considered in connection with the behavior of the real states. Here we consider as real states those corresponding in either sense, a.e. or Filippov, to feedback control laws in (2.1), in order to describe any real behavior in a more direct and realistic way than in previous works.

We remark that the uniqueness condition (A) is fulfilled provided there exists an unique equivalent control (see [3]), as quite often it happens in the applications (see [14]).

Given $x \in C^{0}([0, T])$, we consider

$$
I(x)=\int_{0}^{T}|s(t, x)| \mathrm{d} t
$$

where $|\cdot|$ is any fixed norm of $R^{M}$. Given $\bar{x} \in R^{N}$, the global optimization problem we consider, denoted by $(W(\bar{x}), I)$, consists of globally minimizing $I$ over the set $W(\bar{x})$ of all state trajectories of (2.1) issued from $\bar{x}$. The problem $(W(\bar{x}), I)$ will be called Tikhonov well-posed with value 0 if the following conditions (C), (D) are met.

(C) There exists a unique $y \in W(\bar{x})$ such that $I(y)=0$.

(D) For every sequence $z_{n}$ such that

$$
\dot{z}_{n}=f\left(t, z_{n}, u_{n}\right) \text { on }[0, T], z_{n}(0)=\bar{x}
$$

corresponding to some sequence $u_{n} \in U_{\infty}$ with sup $\left\|u_{n}\left[\cdot, z_{n}(\cdot)\right]\right\|_{\infty}<+\infty$, if $I\left(z_{n}\right) \rightarrow 0$ then $z_{n} \hookrightarrow y$.

Of course, $y=\arg \min (W(\bar{x}), I)$ by $(\mathrm{C})$. Every sequence $z_{n}$ as in (D) will be referred to as a minimizing sequence of $(W(\bar{x}), I)$. 
We shall employ in the sequel the following assumptions.

For every compact set $S \subset U$ there exist $A, B \in L^{1}(0, T)$ such that

$$
|f(t, x, u)| \leq A(t)|x|+B(t)
$$

for a.e. $t$, every $u \in S$ and $x \in R^{N}$.

For every compact $Z \subset R^{N} \times U$ there exists $C \in L^{1}(0, T)$ such that

$$
\left|f\left(t, x^{\prime \prime}, u\right)-f\left(t, x^{\prime}, u\right)\right| \leq C(t)\left|x^{\prime \prime}-x^{\prime}\right|
$$

for a.e. $t$, every $\left(x^{\prime \prime}, u\right),\left(x^{\prime}, u\right) \in Z$.

If $U$ is compact and (2.3) holds, it follows by Gronwall's lemma and standard properties of Filippov solutions that $U_{\infty}$ contains all Carathéodory's feedback controls $u=u(t, x)$, and more generally those which are $L \otimes B$-measurable, see $[7,10]$.

In the following we denote by co the closed convex hull, and by $B(z, \delta)$ the closed ball of radius $\delta$ around $z$.

\section{Approximability AND Well-Posedness}

In this section we prove that approximability of the control system (2.1), (2.2), and Tikhonov well-posedness of $(W(\bar{x}), I)$, are equivalent properties.

Theorem 3.1. Suppose that (2.3) and (2.4) hold. Then $(W(\bar{x}), I)$ is Tikhonov well-posed with value 0 for every $\bar{x} \in R^{N}$ such that $s(0, \bar{x})=0$ if and only if the control system (2.1), (2.2) fulfills the approximability property.

Proof. We show that well-posedness implies approximability. Given $\bar{x}$ and a sequence $\left(u_{n}, x_{n}\right)$ as in condition (B), consider $z_{n}$ such that

$$
\dot{z}_{n}=f\left(t, z_{n}, u_{n}\right) \text { on }[0, T], z_{n}(0)=\bar{x},
$$

in the following sense. If $x_{n}$ is an a.e. (respectively, a Filippov) solution, then $z_{n}$ is an a.e. (respectively, a Filippov) solution. Since $u_{n} \in U_{\infty}$, every $z_{n}$ is defined on the whole $[0, T]$ by $(2.3)$ and $z_{n} \in W(\bar{x})$, see Theorem 5.2 , p. 58 of [7]. Now let $z_{n}$ be a Filippov solution for infinitely many $n$. Fix any subsequence of $z_{n}$ (we do not relabel). Then by the definition of Filippov solution [10] (p. 202), for every $n$

$$
\dot{z}_{n}(t) \in \operatorname{cog} g_{n}\left[t, B\left(z_{n}(t), \delta\right)\right]
$$

for a.e. $t$ and every $\delta>0$, where

$$
g_{n}(t, x)=f\left[t, x, u_{n}(t, x)\right] .
$$

It follows that, by Carathéodory's convexity theorem, $\dot{z}_{n}(t)$ is almost everywhere a limit of points

$$
\sum_{i=1}^{N+1} \alpha_{i}(t) g_{n}\left[t, q_{i}(t)\right]
$$

where $\left|q_{i}(t)-z_{n}(t)\right| \leq \delta$ and $\alpha_{i}(t) \geq 0, \sum_{i=1}^{N+1} \alpha_{i}(t)=1$. Then by (2.3)

$$
\begin{aligned}
\left|g_{n}\left[t, q_{i}(t)\right]\right| & \leq A(t)\left|q_{i}(t)-z_{n}(t)\right|+A(t)\left|z_{n}(t)\right|+B(t) \\
& \leq\left(\delta+\left|z_{n}(t)\right|\right) A(t)+B(t) .
\end{aligned}
$$


Hence by the convergence of the points in (3.1)

$$
\left|\dot{z}_{n}(t)\right| \leq\left(\delta+\left|z_{n}(t)\right|\right) A(t)+B(t)
$$

whence, letting $\delta \rightarrow 0$,

$$
\left|z_{n}(t)\right| \leq \text { constant }+\int_{0}^{t}\left[A(s)\left|z_{n}(s)\right|+B(s)\right] \mathrm{d} s .
$$

It follows by Gronwall's lemma that $z_{n}$ is equibounded. Then we apply Theorem 9, p. 216 of [10] (taking into account (2.3) and equiboundedness), thus we get

$$
x_{n}-z_{n} \hookrightarrow 0
$$

Since $x_{n}$ and $z_{n}$ are equibounded and $s$ is uniformly continuous on compact sets, we obtain $s\left(t, z_{n}\right) \hookrightarrow 0$. It follows that $z_{n}$ is a minimizing sequence of $(W(\bar{x}), I)$, hence

$$
z_{n} \hookrightarrow y=\arg \min (W(\bar{x}), I)
$$

by well-posedness. Then we get

$$
x_{n} \hookrightarrow y
$$

for the corresponding subsequence. Now let $z_{n}$ be an a.e. solution for infinitely many $n$. Then Gronwall's lemma again implies (3.3). The same conclusion holds if $z_{n}$ is an a.e. solution for infinitely many $n$ and a Filippov solutions for infinitely many $n$. Summarizing, in each case uniqueness of the sliding state yields (3.4) for the whole sequence, thereby proving approximability.

Now we show that approximability implies well-posedness. Given $\bar{x}$ let $x_{n}$ be any minimizing sequence of $(W(\bar{x}), I)$. Let $x_{n}$ be an a.e. solution for infinitely many $n$. Then by $(2.3),(2.4)$ and Gronwall's lemma we get in a standard way that $x_{n}$ is equibounded and equicontinuous. Let $x_{n}$ be a Filippov solution for infinitely many $n$. By (3.3) written for $x_{n}$, for each $t^{\prime}, t^{\prime \prime}$ in $(0, T)$ with $t^{\prime}<t^{\prime \prime}$ we have

$$
\left|x_{n}\left(t^{\prime \prime}\right)-x_{n}\left(t^{\prime}\right)\right| \leq \int_{t^{\prime}}^{t^{\prime \prime}}\left[A(t)\left|x_{n}(t)\right|+B(t)\right] \mathrm{d} t
$$

yielding equicontinuity of $x_{n}$ (because of equiboundedness). In every case, fix any subsequence of $x_{n}$ (we do not relabel), then a further subsequence $x_{n} \hookrightarrow z$ by Ascoli-Arzelá's theorem, where $z \in C^{0}([0, T])$ and $z(0)=\bar{x}$. Since $s\left[\cdot, x_{n}(\cdot)\right] \rightarrow 0$ in $L^{1}(0, T)$, for a further subsequence we have $s\left[\cdot, x_{n}(\cdot)\right] \rightarrow 0$ a.e. in $(0, T)$, hence

$$
s\left[\cdot, x_{n}(\cdot)\right] \hookrightarrow s[\cdot, z(\cdot)],
$$

whence

$$
s[t, z(t)]=0 \text { for all } t \in[0, T]
$$

Approximability then implies

$$
x_{n} \hookrightarrow y
$$

the unique sliding state issued from $\bar{x}$. The subsequence we started from was arbitrarily fixed, hence, by the uniqueness of $y$, the whole sequence

$$
x_{n} \hookrightarrow y=\arg \min (W(\bar{x}), I),
$$

whence well-posedness. 


\section{Approximability CRIteria}

Making use of the variational interpretation of approximability we obtain three new criteria, as follows.

Corollary 4.1. The control system (2.1), (2.2) fulfills the approximability property if Conditions (2.3), (2.4) are met, $U$ is compact, $f(t, x, U)$ is convex for a.e. $t$ and every $x$ with $s(t, x)=0$, and for every $\bar{x} \in R^{N}$ with $s(0, \bar{x})=0$ there exists a unique sliding state issued from $\bar{x}$.

Proof. By Theorem 3.1 it suffices to show Tikhonov well-posedness of $(W(\bar{x}), I)$. Let $x_{n}$ be any minimizing sequence. Then

$$
\dot{x}_{n}(t) \in f\left(t, x_{n}(t), U\right) \text { a.e. }
$$

if $x_{n}$ is an a.e. solution. If $x_{n}$ is a Filippov solution, then by the definition (see [11])

$$
\dot{x}_{n}(t) \in \operatorname{co} f\left(t, B\left(x_{n}(t), \delta\right), U\right),
$$

for a.e. $t$ and every $\delta$. In both cases, writing

$$
F(t, y, \delta)=\operatorname{co} f(t, B(y, \delta), U)
$$

we get

$$
\dot{x}_{n}(t) \in F\left(t, x_{n}(t), \delta\right)
$$

for a.e. $t$ and every $\delta>0$. As in the proof of Theorem 3.1, $x_{n}$ is equibounded. Then if $\left|x_{n}(t)\right| \leq M$ for all $t$ and $n$, by (3.2) written for $x_{n}$ we have

$$
\left|\dot{x}_{n}(t)\right| \leq M A(t)+B(t)
$$

for every $n$ and a.e. $t$. Given $\delta>0$, the set-valued map

$$
(t, y) \rightarrow F(t, y, \delta), 0 \leq t \leq T,|y| \leq M
$$

is integrably bounded by (2.3), it takes compact convex values and it is measurable with respect to $t$ for each $y$. Since $f(t, \cdot, \cdot)$ is continuous, the set-valued map

$$
y \rightarrow f(t, B(y, \delta), U)
$$

is upper-semicontinuous [1] (Sect. 1.4), hence the same holds for $F(t, \cdot, \delta)$ for each $t, \delta$. We are in position to apply Theorem 3.1.7 of [5]. It follows that some subsequence (we do not relabel) $x_{n} \hookrightarrow z$ where $z$ is absolutely continuous and by $(4.1)$

$$
\dot{z}(t) \in F(t, z(t), \delta)
$$

for a.e. $t$ and every $\delta$. By upper semicontinuity of $F(t, z(t), \cdot)$ [1] (Sect. 1.4) it follows that

$$
\dot{z}(t) \in \operatorname{co} f(t, z(t), U)
$$

a.e. in $(0, T)$. Since $x_{n}$ is a minimizing sequence we have

$$
s\left[\cdot, x_{n}(\cdot)\right] \rightarrow 0 \text { in } L^{1}(0, T)
$$


hence by uniform convergence

$$
s[t, z(t)]=0,0 \leq t \leq T .
$$

Then by (4.2), (4.3) and the convexity assumption we obtain

$$
\dot{z}(t) \in f(t, z(t), U)
$$

a.e. in $(0, T)$. We apply the measurable selection theorem to the multifunction $\Gamma$ given by

$$
\Gamma(t)=\{v \in U: \dot{z}(t)=f(t, z(t), v)\}
$$

a.e. $t \in(0, T)$ (see [13], Th. 2J). Then there exists a measurable function $u$ such that $u(t) \in U$ and $\dot{z}(t)=$ $f[t, z(t), u(t)]$ a.e. in $(0, T)$. Since $U$ is compact, $z \in W(\bar{x})$. Then by $(3.4), z=y$ the unique sliding state issued from $\bar{x}$. It follows that the original sequence $x_{n} \hookrightarrow y=\arg \min (W(\bar{x}), I)$, hence well-posedness.

Remark 4.1. Compactness of $U$ can be avoided in Corollary 4.1 provided we assume convexity of all sets $f(t, x, D), D$ any compact subset of $U$.

Remark 4.2. Corollary 4.1 generalizes some approximability conditions proved in [3,4]. In [3], approximability is shown to hold provided the plant is affine in the control variable, we have existence and uniqueness of the equivalent control, and some nonsingularity conditions are met. No such conditions are required in Corollary 4.1. Of course, if $U$ is convex, affinity of $f(t, x, \cdot)$ implies convexity of $f(t, x, U)$. In [4], a notion of generalized approximability is shown to hold provided the convexity condition is fulfilled, there exists a unique sliding state and $s=s(x)$ only. In Corollary 4.1 we let $s$ depend of $t$ as well.

According to the terminology of [8], I.2, a function

$$
c: D \rightarrow[0,+\infty)
$$

is called forcing if

$$
0 \in D \subset[0,+\infty), c(0)=0
$$

and for every sequence $a_{n} \in D$,

$$
c\left(a_{n}\right) \rightarrow 0 \Rightarrow a_{n} \rightarrow 0
$$

Corollary 4.2. Under Conditions (2.3), (2.4), if for every $\bar{x} \in R^{N}$ such that $s(0, \bar{x})=0$ there exist a sliding state $y \in W(\bar{x})$ and a forcing function $c$ such that

$$
\int_{0}^{T}|s(t, x)| \mathrm{d} t \geq c\left(\|x-y\|_{\infty}\right), x \in W(\bar{x}),
$$

then the control system (2.1), (2.2) fulfills the approximability property. The converse holds under the assumptions of Corollary 4.1.

Proof. Condition (4.4) implies well-posedness of every $(W(\bar{x}), I)$ by [8], Theorem 12, p. 6, hence approximability by Theorem 3.1. Conversely, approximability yields well-posedness by Corollary 4.1, hence (4.4) again by [8], Theorem 12, p. 6 .

A further criterion can be obtained from a pointwise version of (4.4). 
Corollary 4.3. The control system (2.1), (2.2) fulfills the approximability property if for every $\bar{x} \in R^{N}$ with $s(0, \bar{x})=0$ there exists a sliding state $y \in W(\bar{x})$; and for every $t \in[0, T]$ there exists a forcing function $c(t, \cdot)$ such that $c(\cdot, a)$ is lower semicontinuous for every $a>0$, and

$$
|s[t, x(t)]| \geq c(t,|x(t)-y(t)|), 0 \leq t \leq T, x \in W(\bar{x}) .
$$

Proof. Let $z \in W(\bar{x})$ be a sliding state, then for every $t \in[0, T]$

$$
0=|s[t, z(t)]| \geq c(t,|z(t)-y(t)|)
$$

hence $z(t)-y(t)=0$ since $c(t, \cdot)$ is forcing. Then the sliding state $y \in W(\bar{x})$ is unique. Let $x_{n} \in W(\bar{x})$ be such that $s\left(\cdot, x_{n}\right) \hookrightarrow 0$. Then $c\left(\cdot,\left|x_{n}(\cdot)-y(\cdot)\right|\right) \hookrightarrow 0$ as well. Arguing by contradiction, if $\left\|x_{n}-y\right\|_{\infty} \not \supset 0$ there exist $\varepsilon>0$ and a subsequence $z_{n}$ of $x_{n}$ such that $\left\|z_{n}-y\right\|_{\infty} \geq \varepsilon$ for every $n$. Thus we find a sequence $t_{n} \in[0, T]$ such that

$$
\left|z_{n}\left(t_{n}\right)-y\left(t_{n}\right)\right| \geq \varepsilon \text { for every } n
$$

It is known that we can assume $c(t, \cdot)$ increasing for every $t$, see [8], p. 10. Then

$$
c\left(t_{n}, \varepsilon\right) \leq c\left(t_{n},\left|z_{n}\left(t_{n}\right)-y\left(t_{n}\right)\right|\right) \leq \| c\left(\cdot,\left|z_{n}(\cdot)-y(\cdot)\right| \|_{\infty}\right.
$$

and, for a subsequence, $t_{n} \rightarrow t^{*} \in[0, T]$. Thus $c\left(t^{*}, \varepsilon\right)=0$, contradicting the definition of forcing function, hence $x_{n} \hookrightarrow y$ as required.

The variational characterization of approximability obtained in this paper allows us to verify it for sliding mode control systems (2.1), (2.2) by checking the explicit conditions provided by Corollaries 4.1, 4.2 or 4.3. The following is an example of application of Corollary 4.1.

Example 4.1. Let $g: R \rightarrow R$ be a fixed Lipschitz continuous function such that $g(0)=0$. Consider the sliding mode control system

$$
\dot{x}_{1}=g\left(x_{2}\right), \dot{x}_{2}=u(1-u),|u| \leq 1, s(t, x)=x_{2}, 0 \leq t \leq T \text {. }
$$

Here $N=2, M=K=1$. Of course Conditions (2.3), (2.4) hold, and $f(t, x, U)$ is convex for every $t, x$. Given $\bar{x}$ with $\bar{x}_{2}=0$, the unique corresponding sliding state is

$$
y_{1}(t)=\bar{x}_{1}, y_{2}(t)=0,0 \leq t \leq T \text {. }
$$

All assumptions required by Corollary 4.1 are fulfilled, hence approximability holds. We note in passing that the equivalent control does not exist, since the mapping $u \rightarrow u(1-u)$ is not one-to-one on $[-1,1]$.

The following is an example of application of Corollary 4.2 .

Example 4.2. Consider the sliding mode control system

$$
\dot{x}_{1}=\arctan x_{2}, \dot{x}_{2}=u(1-u),|u| \leq 1, s(t, x)=x_{2}, 0 \leq t \leq T \text {. }
$$

Of course (2.3) and (2.4) are fulfilled and, as in Example 4.1, the unique sliding state $y \in W(\bar{x})$, if $\bar{x}_{2}=0$, is given by (4.5). Let us check (4.4). Given $\bar{x}$ with $\bar{x}_{2}=0$, and any $x \in W(\bar{x})$, we compute

$$
\left|x_{1}(t)-\bar{x}_{1}\right|=\left|x_{1}(t)-y_{1}(t)\right| \leq \int_{0}^{t}\left|\arctan x_{2}(\alpha)\right| \mathrm{d} \alpha \leq \int_{0}^{T}\left|x_{2}(\alpha)\right| \mathrm{d} \alpha, 0 \leq t \leq T,
$$


hence

$$
\int_{0}^{T}|s(t, x)| \mathrm{d} t \geq\left\|x_{1}-y_{1}\right\|_{\infty}
$$

Let

$$
Q: W(\bar{x}) \rightarrow C^{0}([0, T]) \text { given by } Q(x)=x_{1},
$$

obviously an uniformly continuous function. If $x^{\prime}, x^{\prime \prime} \in W(\bar{x})$ and $Q\left(x^{\prime}\right)=Q\left(x^{\prime \prime}\right)$ we have

$$
\arctan x_{2}^{\prime}(t)=\arctan x_{2}^{\prime \prime}(t) \text { a.e. in }(0, T)
$$

hence $x_{2}^{\prime}=x_{2}^{\prime \prime}$, whence $x^{\prime}=x^{\prime \prime}$. Thus $Q$ is one-to-one. For any continuous vector $z$ on $[0, T]$ with components $z_{1}, z_{2}$ we write

$$
\|z\|_{\infty}=\max \left\{\left|z_{1}(t)\right|: 0 \leq t \leq T\right\}+\max \left\{\left|z_{2}(t)\right|: 0 \leq t \leq T\right\} .
$$

Let $\delta$ be the modulus of uniform continuity of $Q$, namely

$$
\delta(\varepsilon)=\sup \left\{\left\|x^{\prime}-x^{\prime \prime}\right\|_{\infty}: x^{\prime}, x^{\prime \prime} \in W(\bar{x}),\left\|Q\left(x^{\prime}\right)-Q\left(x^{\prime \prime}\right)\right\|_{\infty} \leq \varepsilon\right\}, \varepsilon \geq 0 .
$$

Then $\delta(0)=0$ since $Q$ is one-to-one, $\delta(\varepsilon) \geq 0$ and $\delta$ is increasing. Now consider

$$
\omega(\varepsilon)=\varepsilon+\delta(\varepsilon), \varepsilon \geq 0
$$

Then $\omega(0)=0, \omega$ is strictly increasing, $\omega \geq \delta$ and for every $x^{\prime}, x^{\prime \prime} \in W(\bar{x})$

$$
\left\|x^{\prime}-x^{\prime \prime}\right\|_{\infty} \leq \delta\left(\left\|x_{1}^{\prime}-x_{1}^{\prime \prime}\right\|_{\infty}\right) \leq \omega\left(\left\|x_{1}^{\prime}-x_{1}^{\prime \prime}\right\|_{\infty}\right),
$$

hence

$$
\left\|x_{1}^{\prime}-x_{1}^{\prime \prime}\right\|_{\infty} \geq \omega^{-1}\left(\left\|x^{\prime}-x^{\prime \prime}\right\|_{\infty}\right)
$$

Then by (4.6)

$$
\int_{0}^{T}|s(t, x)| \mathrm{d} t \geq \omega^{-1}\left(\|x-y\|_{\infty}\right), x \in W(\bar{x})
$$

which proves (4.4) since $\omega^{-1}$ is forcing. Then approximability holds by Corollary 4.2.

Remark 4.3. If the optimal control problem $(W(\bar{x}), I)$ has optimal value 0 , then the sliding state issued from $\bar{x}$ is a minimizer of $\int_{0}^{T}|s(t, x)|^{2} \mathrm{~d} t$ on $W(\bar{x})$. However, applying the maximum principle we get no information since the Pontryagin's Hamiltonian

$$
H(t, x, u)=q(t)^{\prime} f(t, x, u)-|s(t, x)|^{2}
$$

$q$ being the adjoint state, is identically 0 along the sliding state.

Acknowledgements. Work supported by MURST, progetto cofinanziato Feedback Control and Optimal Control. A preliminary version of this paper was presented at the workshop on Variational Analysis and Applications, Erice, June 2003. 


\section{REFERENCES}

[1] J.-P. Aubin and H. Frankowska, Set-valued analysis. Birkhäuser (1990).

[2] G. Bartolini, A. Levant, A. Pisano and E. Usai, Higher-order sliding modes for the output-feedback control of nonlinear uncertain systems. Sliding mode control in engineering, W. Perruquetti and J.P. Barbot Eds., Dekker (2002).

[3] G. Bartolini and T. Zolezzi, Control of nonlinear variable structure systems. J. Math. Anal. Appl. 118 (1986) 42-62.

[4] G. Bartolini and T. Zolezzi, Behavior of variable-structure control systems near the sliding manifold. Syst. Control Lett. 21 (1993) 43-48.

[5] F.H. Clarke, Optimization and nonsmooth analysis. Wiley-Interscience (1983).

[6] R.A. DeCarlo, S.H. Zak and G.P. Matthews, Variable structure control of non linear systems: a tutorial. Proc. IEEE 76 (1988) $212-232$.

[7] K. Deimling, Multivalued differential equations. De Gruyter (1992).

[8] A. Dontchev and T. Zolezzi, Well-posed optimization problems, Springer. Lect. Notes Math. 1543 (1993).

[9] C. Edwards and S. Spurgeon, Sliding mode control: theory and applications. Taylor and Francis (1988).

[10] A.F. Filippov, Differential equations with discontinuous right-hand side. Amer. Math. Soc. Transl. 42 (1964) $199-231$.

[11] A.F. Filippov, Differential equations with discontinuous righthand sides. Kluwer (1988).

[12] W. Perruquetti and J.P. Barbot Eds., Sliding mode control in engineering. Dekker (2002).

[13] R.T. Rockafellar, Integral functionals, normal integrands and measurable selections, Springer. Lect. Notes Math. 543 (1976) $157-207$.

[14] V. Utkin, Sliding modes in control and optimization. Springer (1992). 